\title{
Is experience in multi-genre video game playing accompanied by impulsivity?
}

\author{
Elham Azizi ${ }^{1}$, Matthew J Stainer², Larry A Abel ${ }^{1}$ \\ 1. Department of Optometry and Vision Sciences, The University of Melbourne, Australia. \\ 2. School of Applied Psychology, Griffith University, Australia.
}

Corresponding author:

Elham Azizi, Department of Optometry and Vision Sciences, Alice Hoy Building, Level 4, University of Melbourne, 3010, Victoria, Australia. Elham.azizi318@gmail.com

\begin{abstract}
Developing impulsivity has been one of the main concerns thought to arise from the increasing popularity of video gaming. Most of the relevant literature has treated gamers as pure-genre players (i.e. those who play only a specific genre of game). However, it is not clear how impulsivity is associated with different genres of games in multi-genre gamers, given that there is increasing diversity in the games played by individuals. In this study, we compared 33 gamers to 23 non-gamers in a go/no-go task: the Continuous Performance Test (CPT). To evaluate whether or not impulsivity occurs as a trade-off between speed and accuracy, we emphasised fast performance to all participants. Then, to examine the ability to predict impulsivity from game genre-hours, we fitted separate multiple regression models to several dependent variables. As an additional measure, we also compared groups in an antisaccade task. In the CPT, gamers showed a trend towards significantly faster reaction time (RT), accompanied by higher false alarm rate (FAR) and more risk-taking response bias $(\beta)$, suggesting impulsive responses. Interestingly, there was a significant negative correlation between RT and FAR across all participants, suggesting an overall speed-accuracy trade-off strategy, perhaps driven by the emphasis on speed during task instruction. Moreover, time spent on role playing games (RPG) and real-time strategy (RTS) games better predicted FAR and $\beta$ than did time spent on action and puzzle games. In the antisaccade task; however, gamers showed a shorter antisaccade latency but a comparable error rate in comparison with non-gamers. There was no specific game genre which could predict performance in the antisaccade task. Altogether, there was no evidence of oculomotor impulsivity in gamers; however, the CPT results suggested the presence of impulsive responses in gamers, which might be the result of a speed-accuracy trade-off. Furthermore, there was a difference in game genres, with time spent on RPG and RTS games being accompanied by greater probability of impulsive responses. Training studies are required to investigate the causality of different video game genres on the development of impulsivity.
\end{abstract}

Keywords: Video gaming, impulsivity, response inhibition, multi-genre gamers, continuous performance test, antisaccade. 


\section{INTRODUCTION}

Playing video games has become one of the major forms of entertainment around the world. In United States, $65 \%$ of American families are home to at least one person who plays video games 3 hours or more per week and $67 \%$ of households own a device that is used to play video games (Entertainment Software Association, 2017). The increasing popularity of video games has drawn researchers' attention to the consequences of extensive playing on users. One of the concerns in this regard has been the development of impulsivity in video gamers.

Impulsivity refers to a predisposition for unplanned actions with little or no forethought to consequences (Daruna \& Barnes, 1993). Impulsivity is a multidimensional concept that incorporates personality, behavioural and biological components. With regards to behavioural component (what we are examining here), it refers to deficits in response inhibition (Littel et al., 2012). While playing a video game, one is required to rapidly press various buttons (this is particularly the case for fast-paced games, including action and driving games) to shoot or move, and also to be able to inhibit erroneous actions at the right time, to avoid things such as losing lives or shooting at friends by mistake. How

does gaming affect our lives outside of the game? We could argue that such a cognitive task might lead to either a disability at inhibiting inappropriate responses (Decker \& Gay, 2011; Deleuze, Christiaens, Nuyens, \& Billieux, 2017) or an enhancement in executive function and decision making abilities (Metcalf \& Pammer, 2014; Strobach, Frensch, \& Schubert, 2012). There is evidence that playing video games decreases reaction time in tasks beyond video games, such as perceptual decision making (Green, Pouget, \& Bavelier, 2010), switching between tasks (Cain, Landau, \& Shimamura, 2012; Colzato, van Leeuwen, van den Wildenberg, \& Hommel, 2010), visual search (Castel, Pratt, \& Drummond, 2005; Hubert-Wallander, Green, Sugarman, \& Bavelier, 2011; Wu \& Spence, 2013) and information processing (Dye, Green, \& Bavelier, 2009b). Therefore, the question arises whether this faster reaction time occurs at the cost of less accuracy and thus is associated with impulsivity or alternately with modified executive function.

Impulsivity in video gamers has been investigated in studies using a variety of behavioural techniques. Colzato, van den Wildenberg, Zmigrod, and Hommel (2013) compared action video gamers with non-gamers in a stop signal task and observed comparable performance between groups on their stop signal reaction time and 
percentage of errors. In line with this study, Metcalf and Pammer (2014) found no evidence of impulsivity in first person shooter gamers (FPS) in comparison with nongamers in either error rate or reaction time (RT) in both a go/no-go and a continuous performance test (CPT). In the first half of the CPT, participants were asked to press the button when the letter " $X$ " appeared on the screen and in the second half whenever the letter " $X$ " appeared following the letter "A". The authors also showed evidence of better decision-making abilities in the lowa gambling task in a FPS gamer group. Dye et al. (2009b) also compared action gamers and non-gamers in the Test of Variables of Attention (TOVA). The test requires the participant to make a quick response to a stimulus appearing in one location (targets), while suppressing a response if it appears at another location (non-targets). In different parts of the test the target can appear either often or very rarely. Examining RT and response sensitivity or d' (which measures the rate of deterioration in performance over time, obtained by calculating the ratio of hit rate to false alarm rate) in the impulsivity condition of the TOVA, they found faster responses in action gamers, who still maintained comparable levels of response sensitivity. Moreover, they conducted a meta-analysis on nine previously published experiments comparing action gamers and non-gamers on speeded choice tasks. They found that action gamers were $11 \%$ faster than non-gamers, although the accuracy level was not different between groups. Altogether, they concluded that there was no evidence of impulsivity in action gamers, in spite of them being faster. Another piece of evidence came from Mack and Ilg (2014), who used an antisaccade task (Hallett, 1978), to test impulsivity between gamers and non-gamers (they did not recruit a specific genre). They showed a faster saccadic reaction time in gamers while the antisaccade error rate was not different between groups, attributing this to there being no difference in impulsivity between gamers and non-gamers (Mack \& llg, 2014).

On the other hand, there is contrasting evidence for impulsivity in video gamers (Decker \& Gay, 2011; Deleuze et al., 2017; Gentile, Swing, Lim, \& Khoo, 2012). Deleuze et al. (2017) examined 81 gamers in three groups: 1) players of online FPS games, 2) multiplayer online battle arena and 3) multiplayer online role playing games, in a behavioural task which served to measure two distinct components of inhibition: cancellation and restraint. They observed a faster reaction time in FPS gamers as well as reduced inhibition abilities in the cancellation task, although not in the restraint task, in comparison to the participants in the other groups. Decker and Gay (2011) also 
compared twelve World of Warcraft players with thirty non-gamers in two versions of a computer-based go/no-go task. The gaming group had faster reaction times and better ability to discriminate between target and distractor (high d'), but showed lower response bias ( $\mathrm{C}$ index, measured by using a signal detection analysis of hit rate and false alarm rate, representing the tendency to respond to both targets and distractors) in both versions of the task. The authors explained this as a pattern of enhanced cognitive function which led to more accurate and faster performance, in spite of being more impulsive. A reduced ability to inhibit a response (i.e., impulsivity) might result in a tendency to respond to all stimuli (i.e., a low $C$ index).

Excessive video gaming (4.67 hours per day for an average of 5 days a week) has also been accompanied by higher levels of self-reported impulsivity (measured by the Eysenck Impulsiveness Questionnaire) as well as lesser inhibition ability, as reflected in a go/no-go task (responding to any letter that appeared on the screen except the one that was similar to the previous one) in comparison to non-gamers. Excessive gamers showed a faster RT in go trials, higher false alarm rate, less sensitivity to detect go and no-go trials and a more risk-taking response bias, indicating their tendency to respond to both go and no-go trials (Littel et al., 2012).

The results obtained are rather ambiguous, with some studies indicating no evidence of impulsivity in gamers, whereas the others showed impulsivity in game players. One potential issue with these studies might be composition of the gamer groups. Gamer groups have mostly been composed of single-genre gamers (those who play a specific genre, with most evidence being available from action games) for at least 4-5 hours a week for a period of time. This classification is somewhat arbitrary, as nowadays most regular gamers tend to play multiple genres rather than playing only a specific one. Recently, Dale and Green (2017) showed that of 5008 individuals who played at least 1.5 hours a week, only $6 \%$ were pure genre gamers (i.e., they played one genre for more than 5 hours/week, but did not play any other genre for more than 3 hours/week), whereas $48 \%$ were multi-genre gamers (i.e., they played more than 5 hours/week, playing more than 3 hours/week of more than one genre) and $47 \%$ were casual gamers (who played between 1.5-5 hours/week). This finding warrants careful consideration of multi-genre playing in video game research if one is to obtain a real view of changes attributed to video gaming. This is an issue with the above-mentioned studies which has 
rarely been acknowledged (but see Dale \& Green, 2017 for mentioning multi-genre gamers ; and Eichenbaum, Kattner, Bradford, Gentile, \& Green, 2015 for including such a classification). Moreover, most of these studies have only investigated action video games and there is scarce data on the other genres in this regard. One possible solution would be looking at game-genre-specific hours that each player allocated to playing a specific genre of game during a week, instead of treating gamers as pure genre gamers. In this way, we would include both multi-genre gamers and single-genre gamers and have a real view of the association between video gaming and impulsivity.

In this study, we aimed to first compare impulsivity between regular video gamers (those who played any video game at least 5 hours a week) and non-gamers, using a go/no-go task: the continuous performance test (CPT). The CPT was initially designed to measure the ability to sustain attention over time (Rosvold, Mirsky, Sarason, Bransome Jr, \& Beck, 1956); however, it also has been used as a measure of impulsivity in the form of motor response inhibition (Riccio, Reynolds, Lowe, \& Moore, 2002). The CPT is a go no-go paradigm where the participant is required to respond with a fast motor response to a frequent go stimulus (target), but to withhold the response when an infrequent no-go stimulus (non-target) is presented. The key variable in this test is the rate of false alarms (i.e. failure to suppress the response to the no-go stimulus). Moreover, to investigate whether or not impulsivity occurs as a result of a trade-off between speed and accuracy, we emphasised to all participants the need for fast performance while performing the CPT task. We then sought to predict impulsivity in gamers from game genre hours via multiple regression analysis.

As an additional measure of impulsivity, we also compared groups in an antisaccade paradigm after they completed the CPT. Antisaccade is considered as a measure of oculomotor impulsivity, although it can account for psychometric trait impulsivity to a limited extent (see Aichert et al., 2012). In this task, participant fixates a central stimulus which will be replaced by a sudden-onset peripheral stimulus. However, the participant is asked to refrain from looking at the peripheral target and instead make a saccade towards the mirror position in the opposite direction (Hallett, 1978). Therefore, it measures the ability to withhold a reflexive prosaccade response to the target, although it is sometimes inefficient when the prosaccade error has already been generated. While 
the task is not directly comparable with the CPT and was not the main focus of the study, it can provide evidence on oculomotor impulsivity.

\section{METHOD}

\section{Participants}

Thirty-three gamers (mean age \pm SD: $22.15 \pm 3.55$ years, 10 females) and twenty-three non-gamers (mean age \pm SD: $23.94 \pm 3.56$ years, 11 females) were recruited either through flyers posted on the university campus or through online advertisements on student noticeboards and staff newsletters at The University of Melbourne. There was no significant difference in the proportion of females and males between groups $(x(1)=0.822$, $p=0.365$ ). Participants who responded to the advertisements were asked to answer a questionnaire via email regarding their demographic details, medical history and previous exposure to games. In the questionnaire, they were asked to report any experience of video game playing during the previous year and, if they had played games, they were asked to name the ten games that they played the most, estimate how long they played each game in a typical session and also how many sessions they played per week (Dye, Green, \& Bavelier, 2009a). Then they were classified as gamers or non-gamers based on their answers and without them being aware of their classification. Participants with zero or less than 1 hour of playing any video games per week over the previous year were considered to be non-gamers. Gamers were chosen from participants who played video games for at least 5 hours a week over the previous year (Hubert-Wallander et al., 2011). Anyone who did not fit these criteria (casual gamers) or reported any neurologic or psychiatric condition did not go through the testing. The gamer group reported an average of $11.12(S D=1.04)$ hours per week of game playing at the time of participation. All participants had normal or corrected to normal visual acuity tested using a LogMAR chart at 3 meters. Participants signed the consent form prior to the testing and received 10 Australian dollars for reimbursement of their time. The study was approved by the University of Melbourne Human Research Ethics Committee.

Gamers in our study played a range of video game genres from first person and third person shooter games (Call of Duty: Modern Warfare 2, Left 4 Dead 2, Counter Strike, Metro 2033, Battlefield 1, Warframe and BioShock Infinite) to action adventure games (Metal Gear Solid, Assassin's Creed 1, 2 \& 3, Last of Us and Star Trek), action role 
playing games (Mass effect 1, $2 \& 3$ and Dead Island), real time strategy games (RTS) (Age of Empires and Warcraft 3: Reign of Chaos), sport games (Pro Evolution Soccer 2013 and FIFA), role playing games (RPG) (Pokemon, Final Fantasy), puzzle games (Tetris, Solitaire) and platform games (Limbo, Portal 2 and Machinarium). 87\% of participants in our sample were multi-genre gamers. According to a video game expertise questionnaire (Green et al., 2017) and a recent commentary by Dale and Green (2017), we classified games into 4 genres including action (first person and third person shooter games, sport games, action adventure and action role playing games), real time strategy (RTS/ multi-player online battle arena), RPG (non-action role playing/ turn-based role playing/ fantasy games) and puzzle games (turn-based strategy, life simulation and puzzle games). Consistent with Dale and Green (2017), we combined action RPG and action adventure games into a single action game group, as they both contain first person or third person combat mechanics as well as the story, explanation and progression of levels typical of RPG and/or adventure games. Sports games were also put into the action genre as they share similar characteristics with shooter games including aiming, shooting, first or third person perspective and real time reaction.

\section{Continuous Performance Test (CPT)}

The Continuous Performance Test was presented using the open access psychological software Psychology Experimental Battery Language Continuous Performance Test (CPT) (version 0.13 , available from http//pebl.sourceforge.net). The CPT consisted of 360 trials. The stimuli were English letters that appeared on the screen one at a time (as one trial) for $250 \mathrm{~ms}$. The trials were presented in 18 consecutive blocks where each block contained interstimulus intervals (ISI) of 1 or 2 or 4 seconds in a random order. Transitions from one block to the next occurred unannounced and without delay. The test was presented on a 20 inch LCD monitor (SAMSUNG, 2043BW, refresh rate $60 \mathrm{~Hz}$ and resolution of $1050 \times 680$ pixels), with participants sitting $50 \mathrm{~cm}$ from the monitor.

\section{Procedure}

The participant's task was to press the spacebar on the keyboard as quickly as possible whenever any letter except " $X$ " appeared on the screen (considered as Go trial) and to withhold their response to the $X$ stimulus (considered as No-Go trial) (Figure 1). Each letter stayed on the screen for $250 \mathrm{~ms}$ and the participant was required to make a 
response to it. Then the next trial began. The target stimuli appeared in $90 \%$ of trials. Participants performed the test in one run, which took 14 minutes to complete.

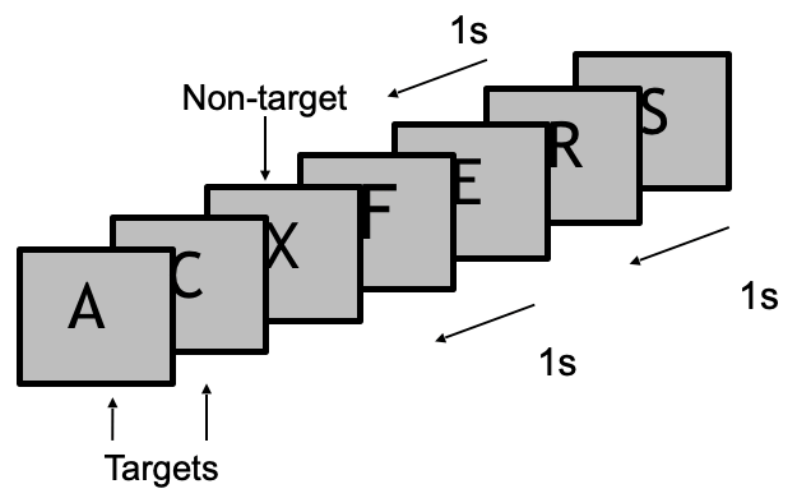

Figure 1: Example sequence of letters in the Continuous Performance Test. Each letter stayed on the screen for $250 \mathrm{~ms}$ with interstimulus intervals of 1 second in this block. After 20 trials, the next block started with ISI of 4 or 2 seconds and so on. Participants were required to make a response to any letter except "X".

Data analyses consisted of the RT in correct trials, false alarm rate (FAR) and response bias $(\beta)$ (see Egeland and Kovalik-Gran $(2010)^{1}$ ). There were other variables, including number of correct trials, target accuracy rate (in percentage), error RT mean, error RT SD and response sensitivity, which were automatically reported by the software but were irrelevant to our analyses. FAR, which is usually used as a measure of disinhibition, shows the proportion of responses to non-target stimulus (i.e. the $X$ in our test). Response bias ( $\beta$ ) describes the tendency to respond to both targets and non-targets. In other words, a participant may be more likely to respond that a target stimulus is present or, alternatively, more likely to respond that a target stimulus is not present. If one tends to respond very cautiously to avoid erroneous responses to the non-target (letter " $X$ " in our test), then the response bias is "conservative", whereas when one responds more freely without concern for errors, the response bias is "risky". Response bias $(\beta)$ in PEBL CPT is calculated based on signal detection theory in

1. They carried out a factor analysis for the variables in Conners CPT (CCPT) for a mix of 376 clinical and normal participants to investigate whether or not the different parameters of the test grouped together relevantly and measured different aspects of attention. They found 5 distinct factors and called them focused attention, impulsivity, sustained attention, vigilance and change in control (see Egeland \& KovalikGran, 2010). 
reporting CPT scores. The formula below shows how the test measures $\beta$ based on hit rate $(H$, the proportion of correct responses to targets) and false alarm rate $(F)$.

$$
\begin{array}{ll}
\beta=\frac{5-4 H}{1+4 F} & \text { if } F \leq 0.5 \leq H ; \\
\beta=\frac{H^{2}+H}{H^{2}+F} & \text { if } F<H<0.5 ; \\
\beta=\frac{(1-F)^{2}+(1-H)}{(1-F)^{2}+(1-F)} & \text { if } 0.5<F<H .
\end{array}
$$

Higher values of $\beta$ indicate a more conservative response, while lower values imply a more risky response bias.

\section{Analysis}

Statistical analyses were carried out using the Statistical Package for the Social Sciences (SPSS, release 2012, IBM SPSS Statistics for Windows, Version 21.0. Armonk, NY: IBM Corp). To compare impulsivity between gamers and non-gamers, we conducted a oneway multivariate analysis of variance (MANOVA) on dependent variables RT, FAR and $\beta$. Furthermore, to predict impulsivity based on genre-specific gaming hours, we evaluated three separate models for each dependent variable using multiple regression analysis.

\section{Antisaccade task}

The same participants carried out antisaccade tasks after they completed the CPT. To record eye movements, we used a $200 \mathrm{~Hz}$ frame rate monocular infrared video eye tracker (SensoMotoric Instruments, Berlin, Germany). Participants sat at $67 \mathrm{~cm}$ from the monitor and their heads were stabilised using a chinrest. Stimuli were presented on a 20 inch LCD monitor (SAMSUNG, 2043BW, refresh rate $60 \mathrm{~Hz}$ and resolution of 1050×680 pixels). A 5-point calibration was conducted before recording eye movements and if the $x$ coordinate spatial accuracy was better than $1^{\circ}$, eye movement recording (left eye) was begun. After calibration, a $1^{\circ}$ centrally positioned black dot was presented for a random time between 1000-2000 ms and then was replaced with a peripheral dot randomly positioned at $5^{\circ}$ or $10^{\circ}$ to the right or left of the centre for a time period between 1000-2000 ms. The peripheral dot then was replaced by a central dot and the next trial 
began. There were 60 trials ( 30 to the right and 30 to the left of the centre) in the experiment, which took about 2 minutes to complete. Participants were instructed to look at the central target. When it was extinguished and replaced with the peripheral target, participants were asked to make an eye movement in the opposite direction but at the same distance. Five practice trials were run to familiarise participants with the task.

Eye movement analysis was performed using a semi-automated program in MATLAB R2010b, (The MathWorks, Inc., Natick, Massachusetts, United States), which allowed exclusion of blinks and other extraneous eye movements. Saccade detection criteria were peak velocity greater than $30 \%$ and acceleration greater than $8000 \% \mathrm{~s}^{2}$. Saccades with latencies shorter than $80 \mathrm{~ms}$ were considered as anticipatory and excluded from the analysis. Median antisaccade latency in correct trials (when the participant successfully inhibited a reflexive saccade and made a saccade in the opposite direction to the target) was calculated for each subject. An antisaccade error was recorded when the initial saccade was made in the same direction as the target, so that trial was considered an error. Unlike in the CPT, every trial required the inhibition of the prepotent response and the concurrent generation of a different response.

\section{RESULTS}

Continuous Performance Test

One participant from the non-gamer group was removed from the analysis as her output contained 49 consecutive missing responses, which was unreliable. One-way MANOVA showed a statistically significant difference between gamers and non-gamers on the linear combination of the three dependent variables $(F(3,51)=5.07, p=0.004$, Wilk's $\Lambda=$ 0.77 , partial $\eta^{2}=0.23$ ). Then to investigate how groups differed on each variable, we looked at tests of between-subject effects.

In the mean reaction time comparison, gamers tended to be faster than non-gamers, although this was just short of significant (355.63 $\pm 35.36 \mathrm{~ms}$ in gamers vs. $377.18 \pm 46.6$ ms in non-gamers) $\left(F(1,53)=3.79, p=0.057\right.$, partial $\left.\eta^{2}=0.067\right)$ (Figure 2$)$.

False alarm rate, however, was significantly different between groups, with gamers showing a higher proportion of incorrect responses to the non-target stimulus $(0.43 \pm$ 
0.17 in gamers vs. $0.33 \pm 0.14$ in non-gamers $)\left(F(1,53)=5.026, p=0.029\right.$, partial $\eta^{2}=$ 0.087) (Figure 2).

Response bias $(\beta)$ also showed a significant difference $(F(1,53)=14.425, p<0.001$, partial $\left.\eta^{2}=0.214\right)$. Gamers had a lower $\beta$ than non-gamers $(0.4 \pm 0.107$ vs. $0.53 \pm 0.14)$, indicating a riskier response bias, as shown in Figure 2. As gamers tended to be faster, showed a riskier response bias and had higher false alarm rates, their performance overall suggested impulsivity.

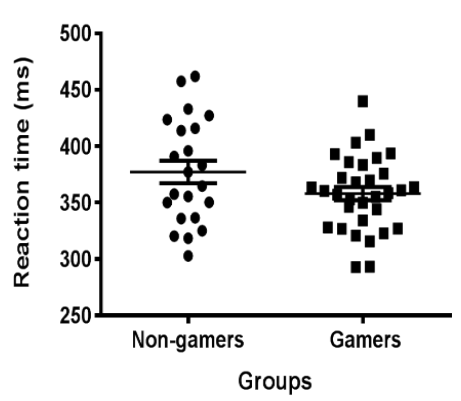

a)

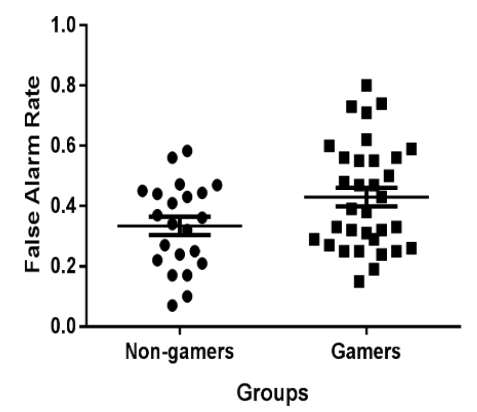

b)

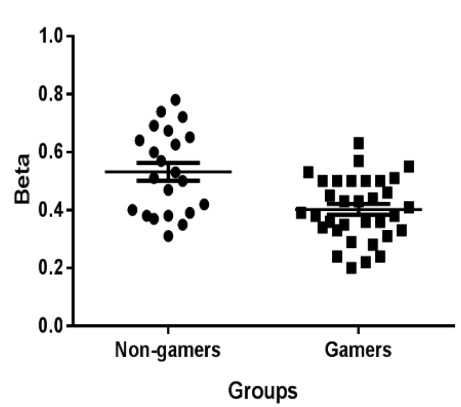

c)

Figure 2: Reaction time (panel a), false alarm rate (panel b) and $\beta$ (panel c) comparison between gamers and non-gamers. Means are indicated by horizontal lines and error bars represent \pm 1 standard error of the mean.

To investigate what linear combination of dependent variables best differentiated gamers from non-gamers, we conducted a discriminant analysis. The standardised discriminant function coefficients were 0.194 for RT, 0.422 for FAR and 1.19 for $\beta$. This indicated that response bias $(\beta)$ had greater discriminating ability to separate groups. The correlation between discriminant function (which is the linear combination of dependent variables) and each variable was 0.955 for response bias, -0.564 for FAR and 0.49 for RT, which again shows the importance of response bias in group classification.

Any evidence of speed-accuracy trade-off?

As video gamers showed faster RTs and higher FARs than non-gamers, it might be inferred that gamers made a speed-accuracy trade-off to perform the task. Hence, we investigated the correlation between RT and FAR across all participants as well as for each group separately. We found a significant negative correlation across all participants $(r=-0.473, p<0.001)$ and for each group separately (gamers: $r=-0.357, p=0.041$, nongamers $r=-0.568, p=0.006$ ) (Figure 3 ). We compared the slopes and intercepts of the 
regression lines using analysis of covariance (ANCOVA) according to Zar (1984). Neither the slopes $(F(1,51)=2.79, p=0.1)$, nor the intercepts $(F(1,52)=1.23, p=0.27)$ were significantly different between groups.

In addition, we used Akaike's Information Criterion (AIC) (in GraphPad Prism 7.03 software) to test whether the data from gamers and non-gamers were better fit by individual curves (for each group) or whether a single curve could be fitted to all data, with "curve" here referring to the linear regressions. The AIC method determines how likely it is that one of two models is the better fit to the data, taking into account both the goodness-of-fit (i.e., sum-of-squares) and the number of parameters in the model (here 2 free parameters for each fit). The results are expressed as the probability that each model is correct, with the probabilities summing to $100 \%$. The AIC calculation showed that the likelihood of one curve giving the best fit for all data sets was $56.64 \%$, whereas the likelihood of one curve for each group being better was $43.36 \%$. The difference between models was small, i.e. -0.534 (the AIC of simple model, i.e. one curve for all data sets minus the AIC of the more complicated model, i.e. one curve for each group). This indicated that either model might be correct and that more data would be needed to settle the question. Therefore, both group regressions estimate the same population regression, indicating the presence of a speed-accuracy trade-off for all participants; however, gamers still made more errors (i.e., had a higher FAR than non-gamers).

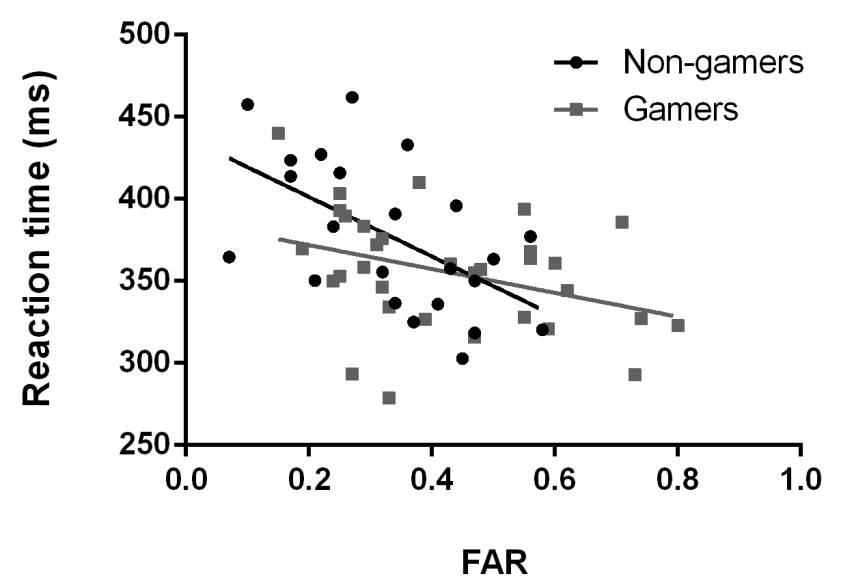

Figure 3: The correlation between RT and FAR for each group separately, FAR= False alarm rate. 
Impulsivity as a function of game genre hours

To investigate whether or not the hours per week that gamers allocated to each genre (action, RTS, RPG and puzzle) could predict impulsivity, we conducted three separate linear multiple regression analyses on the dependent variables.

Multiple regression analysis to predict RT from action, RTS, RPG and puzzle game hours showed that the model was not a good fit to the data $(F(4,28)=0.79, p=0.55), R^{2}=$ 0.174 , adjusted $R^{2}=-0.046$. None of the game genre hours were significant predictors of RT $(p>0.05)$.

However, multiple regression analysis to predict FAR from game genre hours showed that the model was an approximate fit to the data $(F(4,28)=3.008, p=0.052), R^{2}=0.445$, adjusted $R^{2}=0.297$. Of the four game genres, RPG $(\beta=0.53, p=0.023)$ and RTS $(\beta=$ $0.494, p=0.04)$ gaming hours significantly predicted FAR. However, puzzle $(\beta=0.359)$ and action $(\beta=-0.037)$ game hours were not significant predictors of FAR $(p>0.05)$. $\beta$ values are standardised coefficients which have been obtained from multiple regression analysis when all genres are included.

The equation to predict FAR from action, RTS, RPG and puzzle weekly hours is:

$F A R=0.295-\left(0.001^{*}\right.$ Action $)+\left(0.024^{*}\right.$ RTS $)+\left(0.032^{*} R P G\right)+\left(0.034^{*}\right.$ Puzzle $)$ Unstandardised $\beta$ values have been reported for the equation.

We fitted response bias based on game genre hours using the multiple regression analysis. The model was not significant $(F(4,28)=2.31, p=0.105), R^{2}=0.382$, adjusted $R^{2}=0.217$. However, among four predictors, RPG time was found to be a significant predictor for response bias $(\beta=-0.487, p=0.043)$ and RTS approached significant $(\beta=$ $-0.441, p=0.076)$.

Finally, we correlated 4 different game genre hours with each other and the results showed a significant inverse correlation between action and puzzle game hours $(r=$ -0.46, $p=0.039$ ). This might account for the fact that these genre hours did not significantly predict the dependent measures in the multiple regression analysis and they might have obscured each other's effect. Therefore, we further conducted multiple 
regression analysis, removing either puzzle or action games. The results showed puzzle games as an approximate predictor of impulsivity when we removed action games ( $p=0.078$ for FAR and $p=0.087$ for bias) and the model was a good fit to the data for FAR $(F(3,29)=4.26, p=0.022), R^{2}=0.44$, adjusted $R^{2}=0.34$. The model was also significant for the response bias $(F(3,29)=3.28, p=0.048), R^{2}=0.382$, adjusted $R^{2}=0.265$. However, action games were still not a predictor of impulsivity when we removed puzzle games ( $p=0.34$ for FAR and $p=0.357$ for bias) and the models were not significant fits to the data sets.

\section{Antisaccade task}

Three gamers and one non-gamer were excluded from the analysis as they had very light coloured irides that were a problem for the eye tracker. Gamers showed a significantly shorter correct antisaccade latency (mean \pm SD: $0.253 \pm 0.038 \mathrm{~s}$ in gamers vs. $0.28 \pm$ $0.054 \mathrm{~s}$ in non-gamers) $(t(50)=2, p=0.04)$ (Figure 4). However, there was no significant difference between groups in their error rate (mean \pm SD: $25.43 \pm 20.4 \%$ in gamers vs. $29.73 \pm 26.25 \%$ in non-gamers) $(t(50)=0.66, p=0.5)$. To investigate whether there was a relationship between correct antisaccade latency and error rate, we conducted a Pearson correlation analysis. The result showed neither a significant relationship between latency and error rate across all participants $(r=0.232, p=0.097)$, nor for each group separately (gamers: $r=0.065, p=0.73$, non-gamers: $r=0.342, p=0.119$ ). Moreover, four different game genre hours could not predict performance of gamers in antisaccade latency $(F(4,25)=$ $1.82, p=0.18)$ and antisaccade error-rate $(F(4,25)=0.54, p=0.7)$.

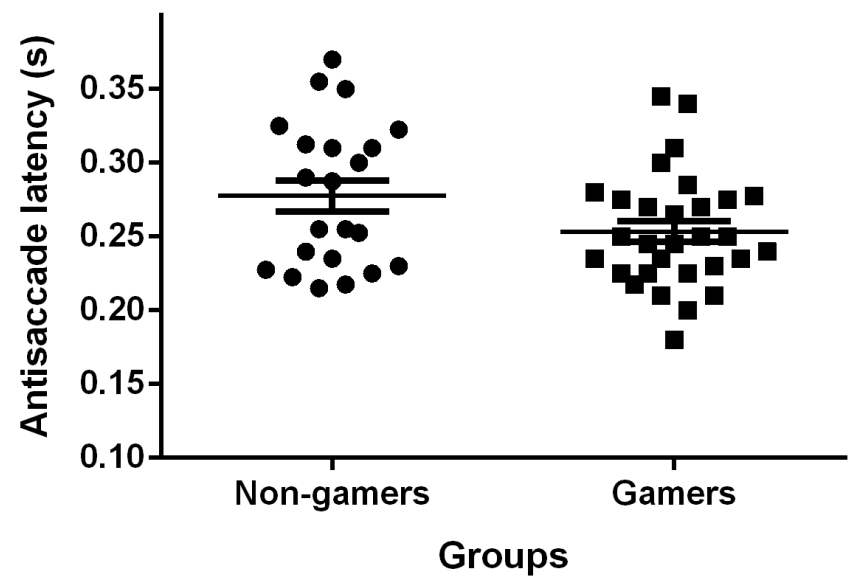

Figure 4: Antisaccade latency (s) comparison in gamers and non-gamers. Means are indicated by horizontal lines and error bars represent \pm 1 standard error of the mean. 


\section{DISCUSSION}

In this study, impulsivity was compared between gamers and non-gamers. In CPT, gamers showed evidence of marginally faster RT, accompanied by a higher false alarm rate and a more risk-taking response bias, all of which suggested impulsivity. We also tested whether or not impulsivity occurred as a consequence of sacrificing accuracy for speed on the CPT task. The results indicated a significant inverse correlation between RT and FAR, not only in gamers but also in non-gamers. This indicates evidence of a speedaccuracy trade-off in all participants including gamers whose impulsivity occurred as a result of their moving further towards the high-speed end of this trade-off.

Gamers were impulsive in comparison to non-gamers in a go no-go CPT; however, there was no evidence of impulsivity in the antisaccade task. We found no difference between groups in their oculomotor response inhibition, in spite of gamers showing shorter antisaccade latencies, thus replicating Mack \& Ilg (2014) findings. These two tasks might not be directly comparable. First, the response modality is different. In the CPT, participants were required to respond by pressing a button, whereas in the antisaccade paradigm, a volitional saccade is required. The inhibition of button presses and saccades might not be equivalent in gamers, as withholding a button press may be more difficult than inhibition of a saccade, possibly due to the common use of button pressing while using game controllers. Moreover, the CPT is a go/no-go task, but there was no analogous element in the antisaccade paradigm as used either by Mack and Ilg (2014) or us. In the antisaccade task, the inhibitory response is essential in each single trial as the subject must withhold a reflexive prosaccade to the peripheral target and then make a correct antisaccade to the opposite direction. In contrast, there is no such requirement to make an inhibitory response in each single trial in the CPT. To investigate the underlying reasons for differences in gamers' performance in the antisaccade and CPT, it would be necessary to make the tasks more comparable, e.g. by adding a go/no-go element to the antisaccade and ensuring the task had the same duration as the CPT. And, finally, It must be noted that not all authors feel that antisaccade paradigm reflects impulsivity; thus, while some authors have considered it as an indicator of impulsivity (e.g. Giel, Schag, Plewnia, \& Zipfel, 2013), others have found that antisaccade error-rate is only modestly correlated with trait impulsivity (e.g. Aichert et al., 2012). 
Gamers in our study showed comparable performance to the excessive gamers in the Littel et al. (2012) study, who had a faster RT, higher FAR and a more lenient response bias compared to control non-gamers or very casual gamers in a go no-go task. The age range was also comparable; however, weekly gaming hours in their excessive gamers were higher than in our gamers. This might be the reason for the higher rates of FAR in this study (54\%), compared to ours $(43 \%)$.

We also found evidence of a speed-accuracy trade-off for all participants in the CPT. This might be because of the task instructions stressing a speeded response. Task instructions and behavioural feedback can shift the strategy of performing a task (Bogacz, Wagenmakers, Forstmann, \& Nieuwenhuis, 2010; Plamondon \& Alimi, 1997). Interestingly, the evidence for this trade-off, the correlation between RT and FAR, was stronger in non-gamers than gamers, although gamers were more impulsive.

Furthermore, to investigate whether game genre hours can predict impulsivity in gamers, we fitted separate regression models for dependent variables. Interestingly, the FAR model was an approximate fit to the data, such that it could predict about $30 \%$ (adjusted $R^{2}$ ) of variability in the data. Among game genres, time spent on RPG and RTS games predicted a greater increase in FAR than time devoted to puzzle and action games. The regression model for response bias also showed that time spent on RPG and RTS were better predictors of response bias than was the time spent on puzzle and action games. However, RPG and RTS game hours were not significantly correlated, indicating that they were separate predictors of impulsivity. Eichenbaum et al. (2015) also found an association between pathological game play (i.e., repetitive use of video games that leads to significant issues in daily life activities such as reductions in academic and professional performance) and genre hours in RPG and RTS games. This might be attributed either to the mechanics of these genres; for example, their particular reward structure, which predisposes players to favour an inferior but instant reward over a large but delayed reward, a mechanism which can elicit impulsive responses (Kim \& Lee, 2011). Alternatively, RPG and RTS players are those who possess specific traits that make them act impulsively. Of course, given the correlational nature of the current study, no inference can be made on the causality of time spent on particular genres of games such as RPG and RTS on impulsivity. However, the study does suggest that not all games have similar effects. Game training studies using these specific genres can 
address this concern. Moreover, in the case of correlational studies, larger sample sizes would allow such models to identify more subtle relationships.

Action genre hours were not a significant predictor of impulsivity (even when puzzle hours were excluded) in the current experiment. This is in line with Metcalf and Pammer (2014), who showed no evidence of impulsivity in FPS gamers, accompanied by better executive function and decision making abilities in an lowa gambling task. Strobach et al. (2012) also demonstrated speeded executive control in "Whether this effect of puzzle gaming is an isolated effect of this sort of game per se or an effect of an interaction of some sort with the other game genres would require further investigation."dual tasking and task switching in action gamers and in non-gamers trained on an action game. It might be related to the action genre features of having situations in which the gamer must assess the risk and benefits and make quick and advantageous decisions, which can improve decision making abilities and enhance executive control processes. Surprisingly, puzzle hours were a predictor of impulsivity when action hours were removed; however, it is not clear why. Whether this effect of puzzle gaming is an isolated effect of this sort of game per se or an effect of an interaction of some sort with the other game genres would require further investigation.

Genre hour classification in this study allowed us to treat multi-gamers with greater detail regarding their gaming activity. However, it needs to be taken into account that the amount of time that each participant said they allocated to each game was always an approximation. It is difficult for participants to accurately recall their gaming history across multiple genres for a year. This, though, is a common problem with self-report measures such as a video game playing questionnaire. One solution suggested by Dale and Green (2017) is to use gaming diaries and to extract game statistics from the game consoles that gamers used.

Taken together, current results cannot confirm whether gamers are individuals who are already impulsive or if playing video games made them so. Gentile et al. (2012) suggested a bidirectional relationship between impulsivity and video game experience, whereby children who are impulsive spend more time playing video games, which in turn subsequently reinforces their attentional problems and impulsiveness. 
Future studies should consider changes in video gaming research in response to ever increasing the popularity of video games, the emergence of multi-genre gamers, the development of games which contain characteristics of more than one genre (hybrid games) and changes in the playing history and demographic of video gamers.

\section{Conclusions}

Current results did not show any evidence of oculomotor impulsivity in gamers; however, there was evidence of impulsive responses (reduced response inhibition) in the go no-go task in gamers, which might occur at the cost of less accurate performance. Moreover, time spent on RPG and RTS games could better predict impulsivity compared with time spent on action and puzzle games. Training studies are needed to prove any causative effect of different video gaming genres on the development of impulsivity.

\section{Acknowledgment}

This work was supported by Albert Shimmins Postgraduate Write-up Award granted to E Azizi.

\section{References}

Aichert, Désirée S, Wöstmann, Nicola M, Costa, Anna, Macare, Christine, Wenig, Johanna R, Möller, Hans-Jürgen, . . . Ettinger, Ulrich. (2012). Associations between trait impulsivity and prepotent response inhibition. Journal of Clinical and Experimental Neuropsychology, 34(10), 1016-1032.

Bogacz, R., Wagenmakers, E. J., Forstmann, B. U., \& Nieuwenhuis, S. (2010). The neural basis of the speed-accuracy tradeoff. Trends in neurosciences, 33(1), 10-16.

Cain, M. S., Landau, A. N., \& Shimamura, A. P. (2012). Action video game experience reduces the cost of switching tasks. Attention, Perception, \& Psychophysics, 74(4), 641-647.

Castel, A. D., Pratt, J., \& Drummond, E. (2005). The effects of action video game experience on the time course of inhibition of return and the efficiency of visual search. Acta Psychologica, 119(2), 217-230.

Colzato, L. S., van den Wildenberg, W. P., Zmigrod, S., \& Hommel, B. (2013). Action video gaming and cognitive control: playing first person shooter games is associated with improvement in working memory but not action inhibition. Psychological Research, 77(2), 234-239.

Colzato, L. S., van Leeuwen, Pieter J. A., van den Wildenberg, Wery P. M., \& Hommel, B. (2010). DOOM'd to Switch: Superior Cognitive Flexibility in Players of First Person Shooter Games. Frontiers In Psychology, 1, 8.

Dale, G., \& Green, C. S. (2017). The Changing Face of Video Games and Video Gamers: Future Directions in the Scientific Study of Video Game Play and Cognitive Performance. Journal of Cognitive Enhancement, 1-15.

Daruna, J. H., \& Barnes, P. A. (1993). A neurodevelopmental view of impulsivity. In The impulsive client: Theory, research, and treatment (pp. 23-37). Washington, DC, US: American Psychological Association.

Decker, S. A., \& Gay, J. N. (2011). Cognitive-bias toward gaming-related words and disinhibition in World of Warcraft gamers. Computers in Human Behavior, 27, 798-810.

Deleuze, J., Christiaens, M., Nuyens, F., \& Billieux, J. (2017). Shoot at First Sight! First Person Shooter Players Display Reduced Reaction Time and Compromised Inhibitory Control in Comparison to Other Video Game Players. Computers in Human Behavior, 72, 570-576. 
Dye, M. W. G., Green, C. S., \& Bavelier, D. (2009a). The development of attention skills in action video game players. Neuropsychologia, 47(8), 1780-1789.

Dye, M. W. G., Green, C. S., \& Bavelier, D. (2009b). Increasing speed of processing with action video games. Current Directions in Psychological Science, 18(6), 321-326.

Egeland, Jens, \& Kovalik-Gran, Iwona. (2010). Measuring Several Aspects of Attention in One Test: The Factor Structure of Conners's Continuous Performance Test. Journal of Attention Disorders, 13(4), 339-346.

Eichenbaum, A., Kattner, F., Bradford, D., Gentile, D. A., \& Green, C. S. (2015). Role-playing and real-time strategy games associated with greater probability of Internet Gaming Disorder. Cyberpsychology, Behavior, and Social Networking, 18(8), 480-485.

Entertainment Software Association. (2017). Essential Facts about the Computer and Video Game Industry.

Gentile, D. A., Swing, E. L., Lim, C, G., \& Khoo, A. (2012). Video game playing, attention problems, and impulsiveness: Evidence of bidirectional causality. Psychology of Popular Media Culture, 1(1), 62-70.

Giel, K. E., Schag, K., Plewnia, C., \& Zipfel, S. (2013). Antisaccadic training to improve impulsivity in binge eating disorder. European eating disorders review, 21(6), 488-492.

Green, C. S., Kattner, F., Eichenbaum, A., Bediou, B., Adams, D. M., Mayer, R. E., \& Bavelier, D. (2017). Playing some video games but not others is related to cognitive abilities: A critique of Unsworth et al.(2015). Psychological Science, 28(5), 679-682.

Green, C. S., Pouget, A., \& Bavelier, D. (2010). Improved probabilistic inference as a general learning mechanism with action video games. Current Biology, 20(17), 1573-1579.

Hallett, PE. (1978). Primary and secondary saccades to goals defined by instructions. Vision Research, 18(10), 1279-1296.

Hubert-Wallander, B., Green, C. S., Sugarman, M., \& Bavelier, D. (2011). Changes in search rate but not in the dynamics of exogenous attention in action videogame players. Attention, Perception, \& Psychophysics, 73(8), 2399-2412.

Kim, Soyoun, \& Lee, Daeyeol. (2011). Prefrontal cortex and impulsive decision making. Biological Psychiatry, 69(12), 1140-1146.

Littel, Marianne, Berg, Ivo, Luijten, Maartje, Rooij, Antonius J, Keemink, Lianne, \& Franken, Ingmar HA. (2012). Error processing and response inhibition in excessive computer game players: an event-related potential study. Addiction Biology, 17(5), 934-947.

Mack, D. J., \& Ilg, U. J. (2014). The effects of video game play on the characteristics of saccadic eye movements. Vision Research, 102, 26-32.

Metcalf, O., \& Pammer, K. (2014). Impulsivity and Related Neuropsychological Features in Regular and Addictive First Person Shooter Gaming. CyberPsychology, Behavior \& Social Networking, 17(3), 147-152.

Plamondon, R., \& Alimi, A. M. (1997). Speed/accuracy trade-offs in target-directed movements. Behavioral and Brain Sciences, 20(02), 279-303.

Riccio, Cynthia A, Reynolds, Cecil R, Lowe, Patricia, \& Moore, Jennifer J. (2002). The continuous performance test: a window on the neural substrates for attention? Archives of Clinical Neuropsychology, 17(3), 235-272.

Rosvold, H Enger, Mirsky, Allan F, Sarason, Irwin, Bransome Jr, Edwin D, \& Beck, Lloyd H. (1956). A continuous performance test of brain damage. Journal of consulting psychology, 20(5), 343.

Strobach, T., Frensch, P. A., \& Schubert, T. (2012). Video game practice optimizes executive control skills in dual-task and task switching situations. Acta Psychologica, 140(1), 13-24.

Wu, Si., \& Spence, I. (2013). Playing shooter and driving videogames improves top-down guidance in visual search. Attention, Perception, \& Psychophysics, 75(4), 673-686. 